\title{
Trabajo Semestral Guiado: un Buen Complemento al Estudio y Aprendizaje en Cursos de Ciencia y de Ingeniería
}

\author{
Alvaro J. Valderrama ${ }^{(1)}$ y José 0 . Valderrama ${ }^{(2)}$ \\ (1) Centro de Información Tecnológica, calle Monseñor Subercaseaux № 667, La Serena - Chile \\ (2) Depto. Ing. Mecánica, Universidad de La Serena, Casilla 554, La Serena-Chile \\ (e-mail: jvalderr@userena.cl)
}

Recibido Dic. 15, 2013; Aceptado Feb. 3, 2014; Versión final recibida Mar. 1, 2014

\begin{abstract}
Resumen
Se describe el desarrollo de un trabajo semestral guiado y se discuten sus ventajas como un buen complemento al estudio y aprendizaje en cursos de ciencia y de ingeniería. Se toma como ejemplo un tema asignado a estudiantes de ingeniería que toman un curso de física en su segundo año de universidad. El artículo detalla primero las ventajas del método de asignación de trabajos guiados, y luego describe paso a paso el desarrollo del proyecto y sus resultados. El ejemplo específico escogido es el estudio del movimiento de un disco volador, conocido como frisbee y la influencia de diversas variables en la trayectoria del disco cuando es lanzado. Este artículo establece que se trata de un trabajo motivador de estudio y aprendizaje, además de otros aspectos formadores del futuro profesional. Desde el punto de vista del estudiante el trabajo muestra que es entretenido y desafiante, ya que son los propios alumnos quienes se fijan el alcance de su proyecto. Los conceptos vertidos en este trabajo pueden ser igualmente aplicables a otros temas de la ciencia y de la ingeniería.
\end{abstract}

Palabras clave: trabajo semestral guiado, estudio y aprendizaje, estudios de física, frisbee

\section{Guided Semester Work: a Good Complement for Studying and Learning in Courses of Science and Engineering}

\begin{abstract}
The development of a guided semester work as a complement for studying and learning in courses of science and engineering is explained. The advantages of this type of semester work are discussed and an example is presented of a semester work given to engineering students in a course in physics. Then the article describes step by step the development of the project and the results. The specific case is the study of the movement of a flying disc, known as Frisbee, and the influence of different variables on the trajectory of the disc when is thrown through the air. The article establishes that the project is a motivating work as a learning tool, besides other aspects of especial importance for the formation of the future professional. From the student's point of view the project shows to be entertaining and challenging, since the students themselves set the scope of the work. The concepts given in this work could be also applicable to other courses of science and engineering.
\end{abstract}

Keywords: guided semester work, studying and learning, physics studies, frisbee 


\section{INTRODUCCIÓN}

Una de las finalidades de la educación y la formación de profesionales es facilitar la comprensión del mundo y la relación con él mediante el uso y la integración de conocimientos. Un trabajo semestral bien guiado como el que se discute en este artículo es una estrategia que permite el fortalecimiento de los procesos cognitivos, además de ir formando en los estudiantes una cultura investigativa, de no solo saber que ciertas cosas suceden sino además interpretar por qué suceden de esa forma. Es en este tipo de trabajos y no en el simple aprendizaje por repetición es donde se manifiesta y cultiva el aprendizaje por descubrimiento, donde los alumnos buscan información que no conocen, la interpretan y la transforman (Vega et al., 2014; Oses y Carrasco, 2013; Hernández et al., 2014). Se trata de una tarea integradora y participativa. Además varios valores para la formación de los estudiantes como individuos integrados a una sociedad pueden ser reforzados en un trabajo bien guiado: responsabilidad en el cumplimiento de las tareas y tolerancia entre cada uno de los integrantes del grupo, por ejemplo. A largo plazo, el futuro profesional podrá desarrollar una capacidad investigadora integral que le permita adaptarse a diversas situaciones en su futura vida profesional, como puede ser por ejemplo abordar temas nuevos dentro de su especialidad (Castellanos y D'Alessandro, 2003).

Desde el punto de vista del estudiante el trabajo semestral bien guiado es entretenido y motivador. En el ejemplo que se presenta en este artículo el alumno elije un tema específico, dentro de un tema global establecido por el profesor. El alumno se preocupa del estudio preliminar que le asegure que cuenta con información suficiente para realizar un buen informe, el alumno integra conocimientos de diversas materias anteriores para tomar decisiones y realizar el trabajo semestral. El trabajo de investigación permite al estudiante establecer contacto con la realidad y constituye un estímulo para la actividad intelectual creadora. Además, ayuda a desarrollar una curiosidad creciente acerca de la solución de problemas y contribuye a fomentar la lectura crítica. Como ejemplo de trabajo semestral bien guiado se presenta y discute en este artículo los resultados de un trabajo asignado en un curso de física de segundo año de Universidad y que el profesor denominó "El Deporte y sus Logros". Las instrucciones dadas por el profesor son bastante detalladas y parten definiendo los objetivos del trabajo, que son dos: i) apreciar la influencia y aplicabilidad de la física en una actividad cotidiana; y ii) familiarizarse con el manejo de magnitudes, unidades y conceptos físicos tales como velocidad, fuerza, impulso, energía, potencia, eficiencia, centro de masas, y momento de inercia, entre otros.

Luego las instrucciones detallan otros aspectos que definen en mejor forma lo que hay que buscar y hacer como contribución original al tema. El largo instructivo se resume en esto: el trabajo de investigación que debe realizar versará sobre un deporte olímpico elegido por el alumno y deberá contener entre otros aspectos una descripción de ellos, sus antecedentes históricos y logros, pero sobre todo, la relación de esos deportes con la ciencia en general y con la física en particular. Además, deberá realizar algún trabajo original sobre algún elemento del deporte. Tales elementos pueden referirse sea a las reglas de esos deportes, como a la indumentaria y otros elementos materiales que se pueden usar en ellos, o a los ambientes en los cuales se practican (Carrasco, 2008).

Las instrucciones también detallan que los alumnos deben entregar Informes de Avance: a) un informe preliminar donde se explica y justifica la elección del deporte o elemento del deporte a estudiar; b) un Informe 1 con los antecedentes históricos y logros del deporte; c) un Informe 2 que incluye análisis del deporte o elemento estudiado; y d) un Informe 3 con los resultados y el aporte del alumno. Los Informes 2 y 3 deben incluir el informe anterior con las correcciones y sugerencias que haya realizado el profesor. Por lo tanto al entregar el Informe 3 se estrega el informe completo del año y corresponde al Informe Final.

\section{INFORME PRELIMINAR}

Se realizó un estudio previo de búsqueda de información para elegir el deporte cuyo análisis se aborda en este trabajo. Esta búsqueda se realizó a través de sitios generales y específicos de deportes en la Internet. En la elección se consideraron aspectos sugeridos en el instructivo dado por el profesor tales como: i) tener acceso a la información pertinente; ii) capacidad de responder a los desafíos con el conocimiento actual y con otros aprendidos en forma particular; y iii) posibilidad de aportar en el estudio del elemento principal del deporte.

Luego de analizar varias opciones (bate de golf, pelota de golf, raqueta de tenis, rueda de una bicicleta, remo de un bote, garrocha), se decidió por el frisbee. Una de las razones es que se ajusta mejor a las materias del curso de físicas en especial velocidad, fuerza, torque. Además se disponía de estudios previos para comparar resultados y hacer una discusión adecuada de la propuesta del trabajo desarrollada durante el semestre. 


\section{INFORME 1: ANTECEDENTES HISTÓRICOS Y LOGROS}

En el trabajo que se discute en este artículo se eligió el "ultimate frisbee" y el principal elemento del juego: el disco. El Disco Volador, denominado Frisbee partió como un simple juguete de jóvenes pero a través del tiempo ha sido usado en varios deportes: ultimate, disco golf, estilo libre, coraje, doble disco. El más popular es el ultimate frisbee o simplemente ultimate, que se ha popularizado en el mundo, pero en particular en Estados Unidos (http://www.wfdf.org/index.php). Hoy existen asociaciones de clubes de ultimate en Europa y Norte América. A altos niveles de competencia, ultimate es un juego intenso, rápido, duro, y que requiere coordinación y concentración de los jugadores. Sin embargo este es un deporte donde se combinan bien la habilidad del deportista y las características del elemento, el disco (Griggs, 2009).

Hay varios estudios en la literatura relacionados con la física del frisbee. Crane (1983) explica algunos detalles cuando frisbees y objetos similares son lanzados a través del aire. La explicación se centra en el balance de fuerzas, en el torque y en el giro del objeto. Nakamura y Fukamachi (1991), estudiaron el flujo alrededor del frisbee visualizándolo como un túnel de viento a baja velocidad. Los autores encontraron que mientras el aire fluye sobre el frisbee se forman un par de vórtices justo detrás del flujo. El vórtice induce suficiente flujo hacia atrás y hacia adelante de modo que se produce una fuerza neta hacia arriba. La rotación del frisbee incrementa también estos flujos y la fuerza hacia arriba. Hummel (2003) describe con detalle la simulación del vuelo de un frisbee y algunos aspectos biomecánicos relacionados con el lanzamiento del frisbee. El autor propone un modelo biomecánico para estudiar con mayor profundidad la cinemática y la cinética del lanzamiento del frisbee. Mungan (2005) discute algunos aspectos relacionados con la enseñanza y la forma de transmitir conceptos relacionados con trabajo y energía. El autor divide su explicación separando el trabajo en dos grandes áreas: trabajo del centro de masa y trabajo de la partícula. Da ejemplos que pueden ser usados en trabajos de grupos en cursos de física. Morrison (2005) visualiza el frisbee como el ala de un avión donde opera el principio de Bernoulli que determina la magnitud de las fuerzas que mantienen al frisbee en el aire, girando y desplazándose. El autor entrega un programa computacional que usa el método simple de Euler para describir la trayectoria del objeto y estudiar la influencia a de diversas variables, tales como fuerza inicial, ángulo de lanzamiento, y velocidad del viento, entre otras. Lorenz (2006) en su libro Vuelos Giratorios describe la dinámica de los boomerangs, frisbees y otros objetos similares. El libro desarrolla el tema desde los conceptos básicos para todo lector hasta detalles técnicos y científicos para ingenieros aeronáuticos.

El frisbee nace a mediados del siglo pasado como un juego de entretención para transformarse años más tarde en un deporte. Durante muchos años el disco ha mantenido un tamaño y masa más o menos definido, aunque los hay en el comercio de distintos materiales, masas y tamaños. Los frisbees originales fueron bandejas de aluminio usadas en unos pasteles muy populares que se vendían en los colegios y universidades en Estados Unidos. La compañía Frisbee Pie Company era la principal proveedora de dichos pastelitos y fueron los estudiantes de la Universidad de Yale quienes los empezaron a usar tirándoselo entre ellos y los denominaron por el nombre que traía impreso, "Frisbee" (Bellis, 2009). En 1981 se fundó la Federación Europea de Disco Volador (EFDF, European Flying Disc Federation, http://www.efdf.org) que daría más tarde origen a la actual Federación Mundial de Disco Volador (WFDF, World Flying Disc Federation, http://www.wfdf.org/index.php) creada en 1984. La Tabla 1 resume los hitos principales en el desarrollo del uso del frisbee y los deportes que lo usan.

Tabla 1: Hitos principales en el desarrollo del uso del frisbee y los deportes que lo usan.

\begin{tabular}{|c|c|}
\hline 1948 & Fred Morrison inventa el frisbee en el año de 1948. \\
\hline $1950-1955$ & Se estudió y confeccionó el reglamento y los niveles de aptitud, en su lugar de origen (California). \\
\hline 1958 & $\begin{array}{l}\text { La compañía Wham-o, una compañía fabricante de juguetes de California (USA) compró los derechos del } \\
\text { disco inventado por Morrison (patente No. D183626 de 1958) y luego registra el nombre de "Frisbee" }\end{array}$ \\
\hline $1960-1966$ & Aquel juego que comenzó por azar se había convertido en un deporte. \\
\hline 1967 & $\begin{array}{l}\text { Se dicta el reglamento y en } 1968 \text { comienza a ser un deporte colectivo y se funda la Federación Olímpica } \\
\text { de Frisbee }\end{array}$ \\
\hline 1970 & $\begin{array}{l}\text { Las primeras reglas que se redactaron para este deporte fueron ideadas por el creador del deporte, Joel } \\
\text { Silver }\end{array}$ \\
\hline 1975 & El primer torneo de ultimate es jugado en el Abril de 1975 en la universidad de Yale. \\
\hline 1979 & $\begin{array}{l}\text { Se forma la asociación de jugadores de ultimate (UPA, Ultimate Players Association). El primer director fue } \\
\text { Tom Kennedy. }\end{array}$ \\
\hline 1981 & Se crea la Federación Europea de Disco Volador (EFDF, European Flying Disc Federation) \\
\hline 1984 & $\begin{array}{l}\text { Se crea la Federación Mundial de Disco Volador (WFDF, World Flying Disc Federation). Los deportes } \\
\text { practicados con discos voladores ya son populares en varios países. }\end{array}$ \\
\hline 1994 & $\begin{array}{l}\text { Se confirma I como miembro regular de la Federación Mundial a la Asociación Mexicana de Disco Volador, } \\
\text { una de las más activas en América. }\end{array}$ \\
\hline
\end{tabular}




\section{INFORME 2: ANÁLISIS FÍSICO DEL FRISBEE VOLANDO}

Aunque la forma del frisbee puede generar levantamiento, no es el único requisito para que el disco vuele. El disco debe girar sobre sí mismo para que pueda mantenerse estable y desplazarse. Los aviones y los pájaros tienen cola para estabilizarse, los misiles tienen aletas. El giro de un frisbee es lo que le da estabilidad. La explicación de porqué es el giro lo que estabiliza a un frisbee es el concepto "momento angular" que se ve en cursos de Física General. De estos cursos se sabe que todo cuerpo que gira tiene "momento angular" y dicho momento puede se puede representar por una magnitud y una dirección (es un vector). El análisis que se hace en este trabajo considera un método discreto de solución donde las diversas variables son analizadas en intervalos pequeños de tiempo. Además, la fuerza (que es un vector) es descompuesta en sus componentes (vertical y horizontal) y lo mismo la velocidad. Y como las componentes en las direcciones $\mathrm{x}$ e y son paralelas, en el análisis que se realiza en este trabajo, las ecuaciones vectoriales se simplifican y se pueden representar mediante expresiones algebraicas.

La ley de conservación del momento indica que el momento angular de un objeto que gira permanece constante a menos que actúe sobre el objeto una fuerza externa. Esto significa por ejemplo que si algo está girando se necesita una fuerza para alterar su eje de rotación (Serway y Faughn, 2004). El Frisbee opera bajo las leyes de mecánica clásica y en su vuelo está sometido a dos fuerzas: la fuerza de elevación y la fuerza de arrastre (Hummel, 2003).

\section{Las fuerzas aerodinámicas influyentes}

Antes de explicar cómo se calculan las fuerzas que actúan sobre el frisbee es necesario conocer los valores de algunos parámetros y variables que aparecen en la descripción matemática que relaciona las fuerzas, la distancia recorrida, y la altura alcanzada por el frisbee. Los datos están tomados de Morrison (2005) y se muestran en la Tabla 2.

\section{Fuerza de arrastre $\left(F_{D}\right)$}

Lo primero para calcular la fuerza de arrastre es calcular el coeficiente de arrastre (CD) que varía en el objeto y se define como una función cuadrática que depende únicamente del ángulo (a) formado entre el plano y el frisbee, considerando los coeficientes $C_{D O}, C_{D a}$ y $\alpha_{o}$ como constantes que dependen de los aspectos físicos del frisbee

$$
C_{D}=C_{D O} \cdot C_{D \alpha} \cdot\left(\alpha-\alpha_{0}\right)^{2}
$$

Luego es necesario calcular el número de Reynolds $(R e=\rho V D / \eta)$ del sistema con el fin de conocer la relación de la fuerza de arrastre aplicada, en este caso tomando los valores de la Tabla 2 como referencia resulta $R e=259 \cdot 10^{5}$; y para un número de Reynolds de tal magnitud la fuerza de arrastre está dada por:

$$
F_{d}=\frac{C_{D} \rho \pi r^{2} v^{2}}{2}=\frac{C_{D} \rho A v^{2}}{2}
$$

\section{Fuerza de elevación $\left(F_{L}\right)$}

La fuerza de elevación es muy similar a la fuerza implicada en un ala de un avión y debido a que el aire es un fluido se calcula utilizando el conocido principio de Bernoulli que da la relación entre la velocidad, la presión y altura de un fluido en cualquier punto de la misma línea de flujo. Los líquidos que fluyen a una velocidad alta tienen una menor presión que los líquidos que fluyen a una velocidad menor. Esto puede ser escrito matemáticamente mediante la ecuación de Bernoulli, con $v$ la velocidad, $p$ la presión, $\rho$ la densidad, $\mathrm{q}$ la aceleración de gravedad y $\mathrm{h}$ la altura respecto del fluido.

$$
\frac{v_{1}^{2}}{2}+\frac{p_{1}}{\rho}+g h_{1}=\frac{v_{2}^{2}}{2}+\frac{p_{2}}{\rho}+g h_{2}
$$

Para este caso en particular la diferencia de altura (el aire que fluye por arriba y el aire que fluye por abajo) es insignificante los términos que dependen de la altura se cancelan. También la velocidad del aire que fluye por encima es directamente proporcional a la que fluye por debajo debido a la diferencia de longitud es constante. Es decir $\left(\mathrm{v}_{1}=\mathrm{C}^{2} \mathrm{v}_{2}{ }^{2}\right)$ obteniendo: 
$\frac{\mathrm{C}^{2} \mathrm{v}_{2}^{2}}{2}+\frac{\mathrm{p}_{1}}{\rho}=\frac{\mathrm{v}_{2}^{2}}{2}+\frac{\mathrm{p}_{2}}{\rho}$

Luego para calcular la fuerza de arrastre es necesario calcular el coeficiente de elevación $\left(\mathrm{C}_{\mathrm{L}}\right)$ que varía en los objetos y se define como una función cuadrática que depende únicamente del ángulo ( $\alpha$ ) formado entre el plano y el frisbee. Considerando los coeficientes $\mathrm{C}_{\mathrm{LO}}, \mathrm{C}_{\mathrm{L} \alpha}$ como constantes que dependen de los aspectos físicos del frisbee, se tiene:

$$
\mathrm{C}_{\mathrm{L}}=\mathrm{C}_{\mathrm{Lo}}+\alpha \mathrm{C}_{\mathrm{L} \alpha}
$$

Tomando en cuenta que $F / A=p_{1}-p_{2}$ (donde $F$ es la fuerza de elevación y $A$ es el área del frisbee), la ecuación queda:

$$
F_{L}=\frac{1}{2} \rho v^{2} A C_{L}
$$

Las diversas fuerzas no se aplican en el centro del disco, pero para mantener el disco volando es necesario un alto momento cinético o angular. Este momento angular se resiste al torque causado por las diversas fuerzas.

Tabla 2: Valores característicos a nivel del mar para un frisbee estándar (aceptado por el consejo nacional de ultimate).

\begin{tabular}{|l|l|l|}
\hline Variable/Propiedad & Unidad & Valor \\
\hline Aceleración de gravedad & $\mathrm{m} / \mathrm{s}^{2}$ & $\mathrm{~g}=-9.81$ \\
\hline Masa del Frisbee & $\mathrm{kg}$ & $\mathrm{m}=0.175$ \\
\hline Densidad del aire & $\mathrm{kg} / \mathrm{m}^{3}$ & $\rho=1.23$ \\
\hline Viscosidad del aire & $\mathrm{Ns} / \mathrm{m}^{2}$ & $\eta=1.73 \cdot 10^{-5}$ \\
\hline Diámetro del Frisbee & $\mathrm{m}$ & $\mathrm{d}=0,26$ \\
\hline Area del Frisbee & $\mathrm{m}^{2}$ & $\mathrm{~A}=0.0568$ \\
\hline Velocidad media al tirar un frisbee & $\mathrm{m} / \mathrm{s}$ & 14 \\
\hline Coeficiente $C_{D O}$ & Adimensional & $\mathrm{C}_{\mathrm{DO}}=0.08$ \\
\hline Coeficiente $C_{D \alpha}$ & Adimensional & $\mathrm{C}_{\mathrm{D} \alpha}=2.72$ \\
\hline Coeficiente $\alpha_{\mathrm{O}}$ & Adimensional & $\mathrm{\alpha}_{\mathrm{O}}=-4$ \\
\hline Coeficiente $C_{\mathrm{LO}}$ & Adimensional & $\mathrm{C}_{\mathrm{LO}}=0.1$ \\
\hline Coeficiente $C_{\mathrm{L} \alpha}$ & Adimensional & $\mathrm{C}_{\mathrm{L} \alpha=1.4}$ \\
\hline
\end{tabular}

\section{Inercia giroscópica}

La rotación del frisbee es un componente necesario para la mecánica de vuelo de un frisbee. Sin rotación el frisbee caería como una hoja al suelo y dejaría de recorrer establemente grandes distancias. Esto se debe a que las fueras aerodinámicas no influyen directamente en el centro del frisbee. En general la elevación en la primera mitad es ligeramente más grande que la segunda mitad de atrás del disco lo que provoca un torque. Las fuerzas y momentos sobre el disco son graficadas claramente por Hummel (2003) y que se reproducen en la Fig. 1.

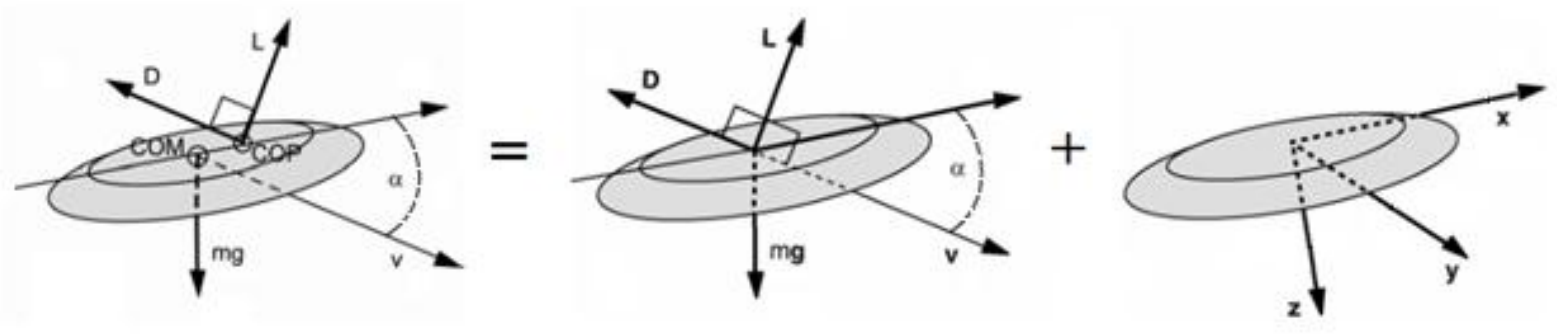

Fig. 1: Fuerzas y momentos sobre el frisbee mostrando el centro de presión (COP), el centro de masa (COM). D es la fuerza de arrastre y L es la de elevación (adaptado de Hummel, 2003) 


\section{Modelo de vuelo del frisbee}

Entre las varias variables que son de interés determinar en el vuelo del frisbee, la distancia horizontal alcanzada, la altura y la precisión son probablemente las de mayor importancia en los juegos con este objeto. Siguiendo los antecedentes de Hummel (2003) y en especial un artículo de Morrison (2005), en este trabajo se ha explorado el efecto de algunas variables del objeto frisbee (masa y diámetro) y del entorno donde se usa (temperatura del aire y altura) sobre la distancia de lanzamiento, uno de las variables de mayor influencia en la práctica de la mayoría de los deportes que usan un disco volador. En el análisis se considera unan variables básicas dadas por Morrison y se varían un 10\% hacia arriba y hacia abajo, para ver su efecto.

El cálculo de las velocidades se puede hacer en forma secuencial y como lo describe Morrison (2005), mediante el método de Euler. En este método se parte de un determinado punto, se fija un rango de tiempo pequeño $(\Delta t)$ y se van calculando las velocidades y distancias en forma secuencial y discreta. Aunque se puede programar en diversos lenguajes, el cálculo se puede hacer también mediante una simple planilla Excel dinámica. El modelo supone que se da suficiente giro inicial para que mantenga un vuelo estable. Si $\Delta \mathrm{v}$ y $\Delta \mathrm{x}$ son cambios en posición y rapidez respectivamente.

$$
\begin{aligned}
& \mathrm{v}_{\mathrm{i}+1}=\mathrm{v}_{\mathrm{i}}+\Delta \mathrm{v} \\
& \mathrm{x}_{\mathrm{i}+1}=\mathrm{x}_{\mathrm{i}}+\Delta \mathrm{x}
\end{aligned}
$$

Considerando los módulos de las fuerzas que actúan vectorialmente, ( $F_{\mathrm{D}}$ : fuerza de arrastre, $\mathrm{F}_{\mathrm{L}}$ : fuerza de elevación, $F_{g}$ : fuerza de gravedad), se obtienen las siguientes ecuaciones para el eje horizontal $x$ :

$$
\begin{aligned}
& \mathrm{F}_{\mathrm{x}}=\mathrm{F}_{\mathrm{D}} \\
& \mathrm{m} \frac{\Delta \mathrm{v}_{\mathrm{x}}}{\Delta \mathrm{t}}=\frac{1}{2} \rho \mathrm{v}_{\mathrm{x}}^{2} A \mathrm{C}_{\mathrm{D}} \\
& \Delta \mathrm{v}_{\mathrm{x}}=\frac{1}{2 \mathrm{~m}} \rho \mathrm{v}_{\mathrm{x}}^{2} A \mathrm{C}_{\mathrm{D}} \Delta \mathrm{t}
\end{aligned}
$$

Y para la vertical y:

$$
\begin{aligned}
& F_{y}=F_{g}+F_{L} \\
& m \frac{\Delta v_{y}}{\Delta t}=m g+\frac{1}{2} \rho v_{x}^{2} A C_{L} \\
& \Delta v_{y}=\left(g+\frac{1}{2 m} \rho v_{x}^{2} A C_{L}\right) \Delta t
\end{aligned}
$$

Usando la ecuación de la rapidez media para el intervalo $\Delta$ t considerado, queda como resultado:

$$
\begin{aligned}
& \Delta x=v_{x} \Delta t \\
& \Delta y=v_{y} \Delta t
\end{aligned}
$$

\section{INFORME 3: APORTE, RESULTADOS Y DISCUSION}

Para este trabajo, se confeccionó un programa en Excel con las ecuaciones dadas por Morrison (2005) y se estudió la influencia de cuatro variables. Los valores base son también los dados por Morrison y que se muestran en las Tablas 3 y 4 . Las figuras 2 y3 muestran algunos resultados: la fig. 2 muestra el efecto del área del frisbee sobre la distancia de lanzamiento, mientras que la figura 3 muestra el efecto de la constante $\mathrm{C}_{\mathrm{do}}$, que depende del material del frisbee, sobre la distancia de lanzamiento. Aunque hay otra serie de resultados, estos se muestran por ser los de mayor importancia, de acuerdo a los cálculos realizados. Las curvas mostradas en las figuras 2 y 3 muestran el comportamiento esperado y están de acuerdo a otros resultados de la literatura (Hummel, 2003; Morrison, 2005). La planilla Excel desarrollada permite obtener las curvas para cualquier conjunto de parámetros y por lo tanto permite hacer otros estudios y aprender más sobre los conceptos de física involucrados. 
Tabla 3: Valores de propiedades del objeto, el disco (Morrison, 2005)

\begin{tabular}{|l|c|c|c|}
\hline Característica del Frisbee & lit & $\min$ & $\max$ \\
\hline Masa del Frisbee $(\mathrm{kg})$ & 0.175 & 0.159 & 0.190 \\
\hline Area del Frisbee $\left(\mathrm{m}^{2}\right)$ & 0.0568 & 0.516 & 0.0625 \\
\hline Coeficiente de empuje en alpha $=0$ & 0.1 & 0.09 & 0.11 \\
\hline Coeficiente de empuje en alpha $\neq 0$ & 1.4 & 1.27 & 1.54 \\
\hline
\end{tabular}

Tabla 4: Valores de variables ambientales usadas en este trabajo (de Astronomía, 2013)

\begin{tabular}{|l|c|c|c|}
\hline Variable del entorno & lit & menor & mayor \\
\hline Aceleración de gravedad $\left(\mathrm{m} / \mathrm{s}^{2}\right)$ & -9.81 & $-9.77($ La Paz, 3720m) & -9.83 (polo sur) \\
\hline Densidad del aire $\left(\mathrm{kg} / \mathrm{m}^{3}\right)$ & 1.23 & $1.20\left(\mathrm{a} 0^{\circ} \mathrm{C}\right)$ & $1.17\left(30^{\circ} \mathrm{C}\right)$ \\
\hline Velocidad del viento $(\mathrm{m} / \mathrm{s})$ & 0 & +1 (favor del viento) & -1 (contra del viento) \\
\hline
\end{tabular}

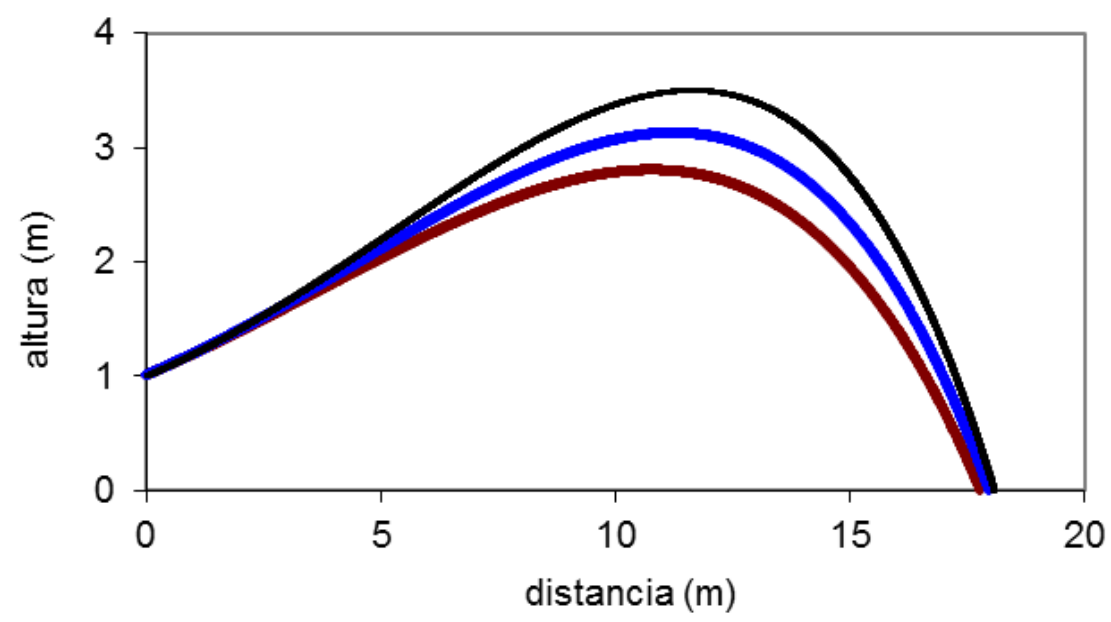

Fig. 2: Efecto del área del frisbee sobre la distancia de lanzamiento. De abajo hacia arriba: $A=0.0516,0.0568$ y $0.0625 \mathrm{~m}^{2}$

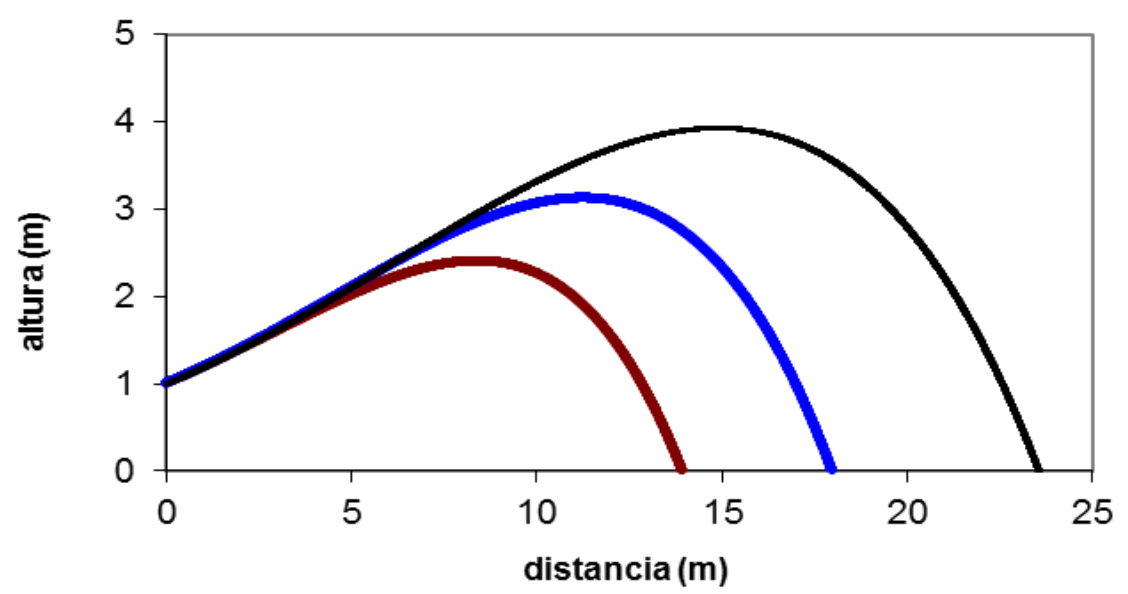

Fig. 3: Efecto de la constante $C_{d o}$, que depende del material del frisbee, sobre la distancia de lanzamiento. De izquierda a derecha: $C_{D_{0}}=0,0.08$ y 0.2 


\section{CONCLUSIONES}

El trabajo semestral resumido en este artículo permite obtener dos conclusiones importantes y que se reflejan en lo expuesto. Una conclusión de tipo técnico basado en los cálculos y una de tipo general basada en el concepto de trabajo guiado.

1) El trabajo semestral bien guiado representa un buen complemento al estudio y aprendizaje en cursos de ciencia y de ingeniería, y en particular al de física general al que corresponde el trabajo desarrollado. Esto se refleja en los numeroso aspectos abordados más allá de las formulas y cálculos que implica el problema abordado.

2) El efecto de las constantes $C_{\text {do }}$ y $C_{\llcorner o}$ es apreciable y dependen de algunas características del material, lo que refuerza la idea de que un mejor frisbee permite obtener mejores rendimientos, distancias y alturas. El área y masa del frisbee tienen más efecto sobre la altura que sobre la distancia, dentro de los márgenes razonables de variación de un frisbee profesional $\left(\mathrm{m}=0.175 \mathrm{~kg}\right.$ y $\left.0.0568 \mathrm{~m}^{2}\right)$.

\section{REFERENCIAS}

Astronomía: http://www.astromia.com/glosario/aceleragravedad.htm (acceso, noviembre 2013)

Bellis M., The First Flight of the Frisbee, http://inventors.about.com/library/weekly/aa980218.htm, página accesada en Agosto (2013).

Carrasco, L.E., El Deporte y sus Logros, trabajo semestral para la asignatura: Física Mecánica Fis 133-3. Pontifica. Univ. Católica de Valparaíso (2008).

Castellanos, M.L. y A. D'Alessandro, Proyectos de Investigación: Una Metodología para el Aprendizaje Significativo de la Física en Educación Media. Rev. Pedagogía, 24(69) 101-136 (2003).

Crane, H. R., Frisbees, can lids, and gyroscopic effects. The Physics Teacher, 21(5) 325-325 (1983)

Griggs, G., The Origins and Development of Ultimate Frisbee. Sport Journal, 12(3) 1-2 (2009).

Hernández, M.R., V.M. Rodríguez, F.J. Parra y P. Velázquez. Las Tecnologías de la Información y la Comunicación (TICs) en la Enseñanza-Aprendizaje de la Química Orgánica a través de Imágenes, Juegos y Videos, 7(1) 31-40 (2014).

Hummel, S.A., Frisbee Flight Simulation and Throw Biomechanics, M. Sc. Thesis, in Mechanical Engineering, University of California, Davis (2003).

Lorenz, R.D., Spinning Flight: Dynamics of Frisbees, Boomerangs, Samaras, and Skipping Stones, Springer, New York (2006).

Morrison, V. R., The Physics of Frisbees. Electronic Journal of Classical Mechanics and Relativity, Mount Allison University, 8(48), 1-12 (2005).

Mungan, C.E. A Primer on Work-Energy Relationships for Introductory Physics. The Physics Teacher, 43(1) 10-16 (2005)

Nakamura Y. y N. Fukamachi, Visualization of the Flow past a Frisbee. Fluid Dynamics Research 7(1) 31-35 (1991).

Oses, S.E. y L.E. Carrasco, Módulos Alternativos en la Enseñanza de las Ciencias. Estrategia Didáctica Orientada al Logro de Aprendizajes Significativos, Formación Universitaria, 6(3) 39-52 (2013).

Serway; R.A. y J.S. Faughn, Fundamentos de Física, vol. 1, Edit. Paraninfo, España, 6ae edición (2004).

Vega, F., E. Portillo, M. Cano y B. Navarrete, Experiencias de Aprendizaje en Ingeniería Química: Diseño, montaje y Puesta en Marcha de una Unidad de Destilación a Escala Laboratorio Mediante el Aprendizaje Basado en Problemas, Formación Universitaria, 7(1) 13-22 (2014). 